\title{
GCU
}

Glasgow Caledonian

University

University for the Common Good

\section{Development of an intelligent robotic system for plasma processing of industrial products with complex shape}

Kussaiyn-Murat, Assel ; Krasavin, Alexander ; Alontseva, Darya ; Kadyroldina, Albina ; Khozhanov, Alexandr ; Krak, lurii ; Munoz de Escalona, Patricia; Dyomina, Irina

Published in:

11th IEEE International Conferences on Intelligent Data Acquisition and Advanced Computing Systems: Technology and Applications (IDAACS'2021)

DOI:

10.1109/IDAACS53288.2021.9660960

Publication date:

2022

Document Version

Author accepted manuscript

Link to publication in ResearchOnline

Citation for published version (Harvard):

Kussaiyn-Murat, A, Krasavin, A, Alontseva, D, Kadyroldina, A, Khozhanov, A, Krak, I, Munoz de Escalona, P \& Dyomina, I 2022, Development of an intelligent robotic system for plasma processing of industrial products with complex shape. in 11th IEEE International Conferences on Intelligent Data Acquisition and Advanced Computing Systems: Technology and Applications (IDAACS'2021). vol. 1, IEEE, pp. 572-579, 11th IEEE International Conference on Intelligent Data Acquisition and Advanced Computing Systems: Technology and Applications , 22/09/21. https://doi.org/10.1109/IDAACS53288.2021.9660960

\section{General rights}

Copyright and moral rights for the publications made accessible in the public portal are retained by the authors and/or other copyright owners and it is a condition of accessing publications that users recognise and abide by the legal requirements associated with these rights.

Take down policy

If you believe that this document breaches copyright please view our takedown policy at https://edshare.gcu.ac.uk/id/eprint/5179 for details of how to contact us. 


\title{
Development of an intelligent robotic system for plasma processing of industrial products with complex shape
}

\author{
Assel Kussaiyn-Murat ${ }^{1 *}$, Alexander Krasavin ${ }^{1}$, Darya Alontseva ${ }^{1}$, Albina Kadyroldina ${ }^{1}$, Alexandr \\ Khozhanov $^{1}$, Iurii $\mathrm{Krak}^{2}$, Patricia Muñoz de Escalona ${ }^{3}$, Irina Dyomina ${ }^{1}$ \\ ${ }^{1}$ D Serikbayev East Kazakhstan Technical University, Ust-Kamenogorsk, Kazakhstan, 070004, Protozanov Street, 69 \\ (e-mail: asselkussaiynmurat@gmail.com; akrassavin@ektu.kz; dalontseva@ektu.kz; akadyroldina@gmail.com; \\ alexskt1@mail.ru; irdyomina@mail.ru) \\ ${ }^{2}$ Taras Shevchenko National University of Kyiv, 64/13 Volodymyrska str., 01601, Ukraine (e-mail: krak@univ.kiev.ua) \\ ${ }^{3}$ Glasgow Caledonian University, Glasgow, United Kingdom, G4 0BA, Cowcaddens Road (e-mail: \\ patricia.munoz@gcu.ac.uk)
}

\begin{abstract}
The paper presents new results of automated path planning for an industrial robot manipulator performing microplasma spraying of coatings on substrates with complex surface shapes. Path planning and automatic generation of the manipulator motion program are performed using data of a preliminary $3 D$ surface scanning by a laser triangulation distance sensor installed on the same robot arm. The automatic manipulator working tool path planning algorithm is based on the choice of the starting segment of the working tool trace as a geodetic line on the surface. An algorithm for optimal spatial curve approximation by a sequence of line segments and arcs has been developed as a part of the automatic manipulator program generation system. The developed algorithms and their software implementation were experimentally tested through robotic microplasma spraying of a protective coating on the surface of a jaw crusher plate, which was then successfully operated for crushing mineral raw materials.
\end{abstract}

Keywords- industrial robot-manipulator, 3D scanning, automatic path planning, automatic robot - manipulator program generation

\section{INTRODUCTION}

Nowadays, industrial robots are widely used, manipulators are used for loading, packaging, plasma or laser cutting and melting as well as for various types of surface treatment: painting, spraying coatings, etc. [1-6]. With regard to the thermal plasma spraying process, the main task performed by the robot arm is to move the plasma source or substrate in order to expose the substrate surface to the plasma flow with the coating particles in a stable, consistent and repeatable manner. This means that during plasma spraying, the robot always maintains the same spraying angle (preferably $90^{\circ}$ ), spraying distance (a distance from the plasma source nozzle to the surface) and fixed distance that the source or substrate is displaced to bring the next section of the surface into the plasma flow $[7,8]$.
From the point of view of the requirements for a uniform distribution of the coating mass on the surface, the plasma spraying process is conducted similar to the processes of paint spraying, where the spraying cone moves along the surface. However, there are significant differences between the processes of plasma spraying and paint spraying, as well as in assessing the quality of the final product (e.g. coating), as will be noted below.

Currently, different approaches are used for a robot arm automatic path and motion planning, i.e. planning the movement of the manipulator working tool along a given spatial trajectory. Trigatti at al. [9] calculated the time parameters of the trajectory of the spray painting robot based on the restrictions of the maximum values of speeds and accelerations of the final effector imposed by both the characteristics of the manipulator and the technological parameters of the spraying process. Chen et al. [1, 10, 11] developed a path planning scheme for a spray painting robot based on the choice of the starting segment of the trajectory as a geodetic line on the sprayed surface. Zhou Yu. et al. [12] emphasized that the shape of the workpiece can strongly influence the quality of the sprayed coating. To simplify the trajectory planning operations for robotic spraying of complex surfaces, Fu et al. [13] proposed a surface segmentation method based on a genetic algorithm, in which a complex surface is divided into several surfaces of small curvatures. Zhou B. et al. [14] developed an algorithm for surface slice-projection processing for automatic generation of the manipulator working tool trajectory, and the simulation results demonstrated the effectiveness of the algorithm. Zeng et al. [15] developed a model of the rate of growth of the coating for the technology of spraying with a different angle of inclination; experiments have shown that the model has good accuracy.

Currently, the use of robotic manipulators in industry is usually limited to large-scale production, since each transition to a new type of product requires complex 
calibration procedures in order to achieve compliance with the model embedded in the robot during its manufacture. Therefore, the task of automatically generating a program code for a robotic arm from a CAD model is in the focus of researchers and developers of robotic systems [9-15]. The practical implementation of such task could make it possible to efficiently process the surfaces of small-scale and piece products of complex shape using a robotmanipulator.

The main idea of this research is to develop an intelligent robotic system for plasma processing of industrial products, which makes it possible to implement the automated pass planning of a robot arm. A distinctive feature of the proposed intelligent robotic system is a preliminary 3D-scanning of the surface of the workpiece with subsequent automatic generation of the robotmanipulator program code, taking into account the data of the 3D-scan of the object (Fig 1). Pre-scanning is carried out by distance sensors mounted on the same robotic arm that plasma sprays the object

Generation of a robot manipulator program for surface scanning

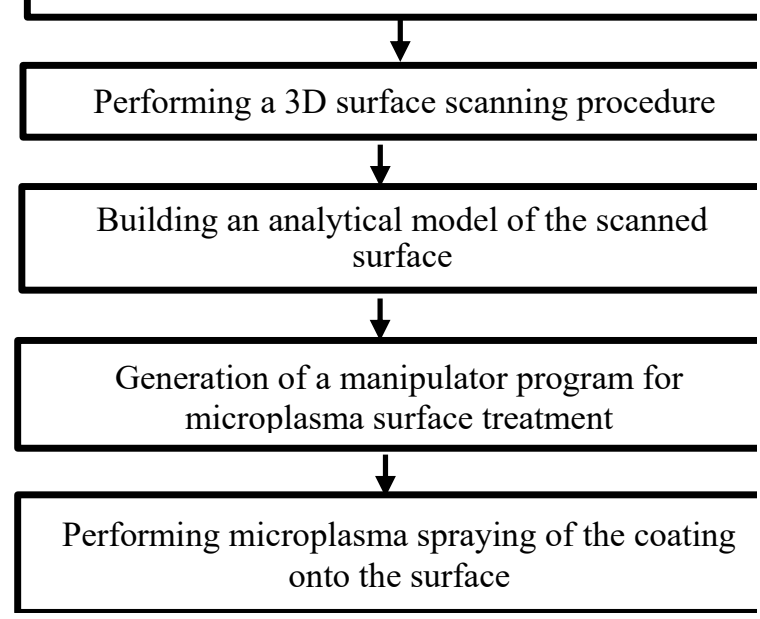

Figure 1. Flow chart of the process of microplasma spraying by an intelligent robotic system

Thus, the trajectory plan must be generated for the thermal spraying process without any initial knowledge of the products shape or orientation. The closest to the main ideas of this study are the different algorithms developed by Kondratenko et al. [16, 17], Tkachenko et al. [18] and Patil et al. [19] for the operation of an intelligent robot in an environment with uncertainty. In this case, the trajectory is formed by the robot control system based on information about the current state of the external environment, that is, according to the 3D model of the processed surface reconstructed by the robot, which is a point cloud (coordinates of the object surface).

The development of such an intelligent robotic system will make it possible to process piece and large-sized products, the geometric parameters of which are determined with low accuracy, or products with deviations from a given shape. At the same time, the method of robotic scanning developed by the authors of this paper is inexpensive compared to machine vision methods, since it uses cheap distance sensors, and it must be highlighted that this method is quite accurate due to the use of an industrial robot with precise positioning of the working tool. To implement the research idea, it was necessary to develop new algorithms (and corresponding software) for scanning and reconstructing a 3D model of an object, including a surface segmentation algorithm, as well as algorithms for automatic generation of a robot code for moving a working tool along the resulting $3 \mathrm{D}$ trajectory.

This study focused on the development of an automatic path and motion planning based on the preliminary 3D surface scanning data for a robot manipulator performing microplasma spraying of coatings on surfaces with complex shapes.

The main purpose of the paper was to develop and test the algorithms for the surface segmentation and automatic generation of a robot manipulator program for a plasma surface treatment of large-sized products. Testing of the intelligent system was carried out in the conditions of the production site for robotic plasma spraying with production tests of plasma sprayed coatings.

\section{EXPERIMENTAL}

The implementation of the automatic path planning based on the 3D model of the scanned object and the motion planning was carried out for the Kawasaki RS010L (Kawasaki Heavy Industries, Japan) robot manipulator controlled by the E40F-A001 programmable controller with the use of AS (Advanced Superior) software [20]. The characteristics of the robotic manipulator Kawasaki RS010LA are as follows: positioning accuracy: $0.06 \mathrm{~mm}$; maximum linear speed: $13100 \mathrm{~mm} / \mathrm{s}$; engagement zone: $1925 \mathrm{~mm}$; working load capacity: $10 \mathrm{~kg}$.

The MPS-004 microplasmatron (E.O. Paton Electric Welding Institute, Ukraine) weighing $1.2 \mathrm{~kg}$ has been mounted on the robot arm and moved to perform microplasma spraying of metal powders onto metal substrates. The speed of linear movement of the microplasmatron along the substrate was chosen to be 50 $\mathrm{mm} / \mathrm{s}$. This speed was chosen experimentally to ensure plasma spraying of the coating with a uniform thickness. Experiments have shown that this speed of linear traveling of the microplasmatron does not lead to disturbances in the plasma jet flow due to air resistance and, therefore, ensures the stability of the spraying process with different parameters [21]. The coating thickness varied from $150 \mu \mathrm{m}$ to $500 \mu \mathrm{m}$ due to changing the plasma spraying parameters (amperage, spraying distance, plasma gas flow rate and powder flow rate) and change in the number of passes of the plasma jet. The main feature of thermal plasma spraying is high temperatures in the plasma jet $(>20.000 \mathrm{~K})$. Powders of refractory metals and alloys or ceramics melt in a plasma jet, deform when hitting the surface, and solidify, forming 
a coating. The main challenge for the development of thermal plasma spraying technologies is the formation of a coating with controlled microstructures and properties. The use of the intelligent robotic systems allowed maintaining the specified speed, spraying distance and angle, providing precision coating deposition on complexshaped implant parts $[21,22]$. It was shown that the robotic microplasma spraying can produce biocompatible coatings on patient with specific medical titanium implants meeting the requirements of international standards of implants for surgery in terms of coating adhesive strength, crystallinity and purity [21]. The small size of the spraying spot on the plasma-coated surface (with a diameter of $5 \mathrm{~mm}$ to $15 \mathrm{~mm}$ ) reduces the loss of spraying material when coating smallsized products, which include most parts of endoprostheses. However, the advantage of the developed intelligent robotic system is that it is applicable for processing largesized objects of complex shape, as will be described below.

The preliminary scanning of substrates was carried out with an ODSL 8/D4-400-S12 (Leuze electronic, Germany) laser triangulation distance sensor installed on the manipulator.

The motion of the robot-manipulator is completely determined by the program in the AS (Advanced Superior) language, executed by the robot's control system. The high level robot-control language AS can be viewed as endeffector motion description language. The AS program describes the motion of the center of the working tool as motion through the number of path positions and also describes spatial orientation of the working tool. High level control software solves reverse kinematic task for given end effector motion description, as well as other trajectory planning tasks. All low level control tasks (such as a force and position control) are performed by the manipulator controller.

\section{RESULTS AND DISCUSSION}

During the scanning procedure, the trajectory of the manipulator working tool (the distance sensor) lies in the horizontal plane above the scanned surface. The trajectory consists of connected U-shaped segments. Work segments of the trajectory are parallel straight line segments that cover the scanned area. When passing the working segments of the trajectory, the distance sensor data is read. The robot manipulator controller allows to read the current spatial position of the working tool on request, and the laser triangulation distance sensor also allows to perform measurement on request. In the process of scanning, a couple of requests are sequentially executed from the computer: i) a request to perform measurements to the distance sensor and ii) a request to transfer the coordinates of the working tool to the controller of the robotic arm. The time interval between these two requests is so small that it is assumed that the distance was measured exactly at the location of the sensor, determined by coordinates received as reply to the second request. The result of scanning procedure is a point cloud. Speaking more precisely, this point cloud is an unordered set of triplets of Cartesian coordinates of points on the scanned surface. For the scanning scheme, described above, the first pair of coordinates (the position of the sensor on the plane of the sensor trajectory) is measured with a high degree of accuracy, while the third coordinate (the distance from the plane of the sensor trajectory to the point of the scanned surface) represents a noisy data of distance sensor. Thus, an attempt to reconstruct the surface by direct methods of 3D will lead to unacceptably large errors.

In order to overcome these difficulties, a data processing algorithm that uses regression analysis methods has been developed in order to build a local parameterizable surface model. The surface segmentation procedure has been described in previous papers [22, 23], during which local models of overlapping surface segments are combined into regions with a homogeneous geometric structure described by a general analytical parameterizable model, which allows without a priori knowledge of the geometric structure surface construct an analytical 3D model of a surface with a complex structure. From authors' knowledge this is the first research team to propose the use of numerical values of the Gaussian and mean curvature of the surface as a criterion for the homogeneous geometric structure of two local regions of the surface [22, 23]. The segmentation procedure is necessary for the further formation of the trajectory of the manipulator for two reasons: i), the methods used to form the path of the manipulator uses an analytical model of the surface as input data and ii) the use of segmentation methods allows to partially solve the problems arising in the reconstruction of surfaces, the shape of which cannot be described by a smooth function of two variables (surfaces with "fractures").

At the first stage of constructing an analytical model of the surface, the coverage of the scanned area with overlapping circles of the same radius was constructed. The centers of the circles formed a rectangular grid. To select geometrically homogeneous areas of the scanned surface, a variation of the region growing algorithm was applied. The region growing algorithm, originally developed for image segmentation, can be generalized for an arbitrary connected graph and in this case, the vertices of the graph can be considered as pixels. So, the input of the algorithm is a graph. The vertices of the graph are grid nodes corresponding to the centers of the circles that define the local approximation regions. The algorithm examines neighboring pixels (vertexes) of initial seed points (vertexes) and determines whether the pixel neighbors should be added to the region. The process is iterated on, in the same manner as general data clustering algorithms. The block diagram of the developed algorithm for constructing a region of homogeneity by the seed point is shown in Fig. 2a. A numerical criterion $\sqrt{\delta_{h}(v)}$ was used to determine whether a neighboring vertex belongs to a region ("Get New Border" in Fig. 2a). To test if a vertex 
$\sqrt{v}$ belongs to region corresponding to a seed point $\sqrt{h}$, the procedure uses the predicate $\sqrt{\delta_{h}}$, defined as (1):

$$
\mid \sqrt{\delta_{h}}=\left\{\begin{array}{l}
1, i f\left|K(v)-K_{0}\right| \prec \varepsilon_{K} \text { and }\left|H(v)-H_{0}\right| \prec \varepsilon_{H}, \\
0, \text { if }\left|K(v)-K_{0}\right| \geq \varepsilon_{K} \text { and }\left|H(v)-H_{0}\right| \geq \varepsilon_{H}
\end{array}\right.
$$

where $\sqrt{K(v)}$ is a value of the Gaussian curvature and $\sqrt{H(v)}$ is a value of the average curvature in the region of local approximation corresponding to a vertex $\sqrt{v}, \sqrt{K_{0}}$ and $\sqrt{H_{0}}$ are curvatures in a local area corresponding to a given seed point (a vertex $\sqrt{h}$ ), $\sqrt{\varepsilon_{K}}$ and $\sqrt{\varepsilon_{H}}$ are the positive thresholds values. The scheme of the seed point's selection is shown in Fig. $2 b$.

The software testing data for this segmentation algorithm written in Python is presented in Fig. 3. The surface elevation map is a segment of a sphere of radius $R=2$, the center of which is located at a point with coordinates $(0,0,-1.5)$, cut by the plane $z=0$. The map is set on a rectangular grid with a step $\Delta x=0.02, \Delta y=0.02$

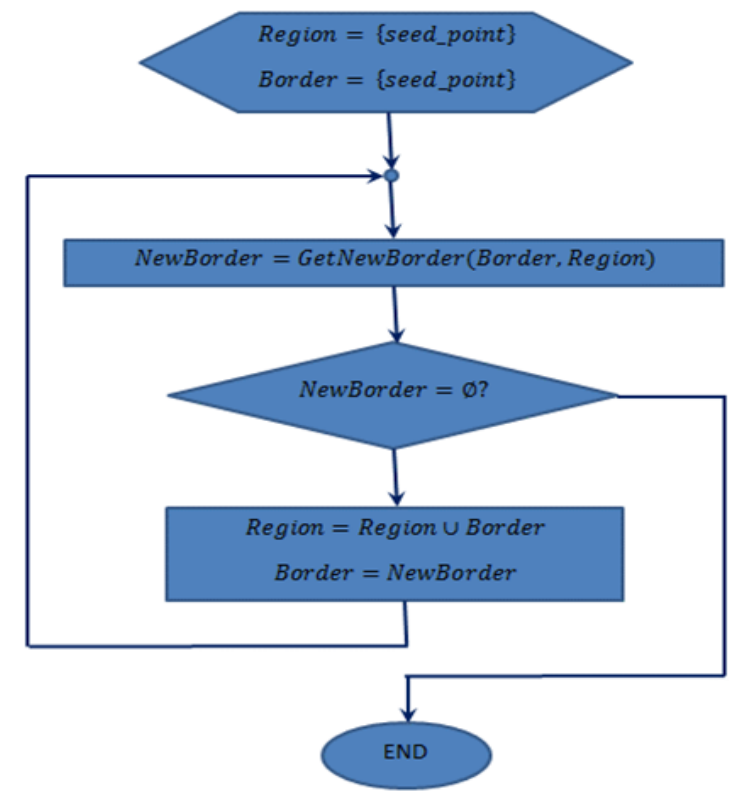

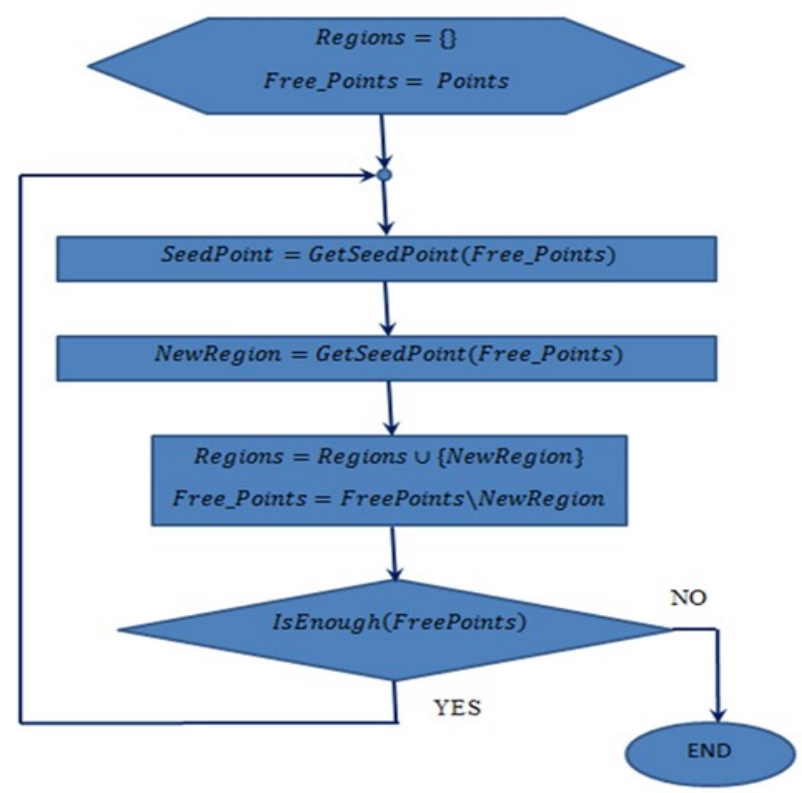

b

a - block diagram of the algorithm for constructing a region of homogeneity by the seed point; $\mathrm{b}$ - block diagram of the algorithm for the seed point's selection

Figure 2. Block diagrams of the surface segmentation algorithms

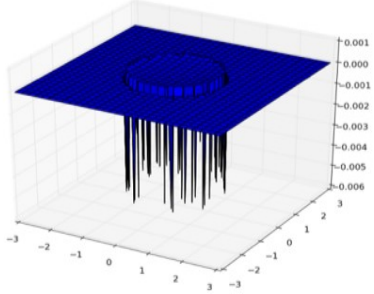

a

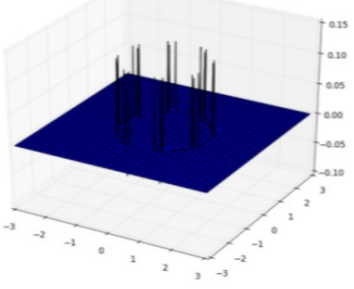

b

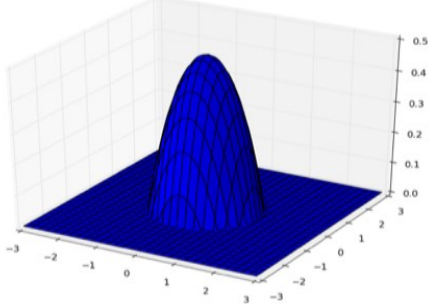

c

a - the surface of the function of the mean curvature of the surface, calculated in local windows of radius $r=0.75$

$\mathrm{b}$ - the surface of the function of the Gaussian curvature of the surface, calculated in local windows of radius $r=0.75$

$\mathrm{c}$ - reconstructed model of the surface - a plane with a segment of a sphere of radius $R=2$

Figure 3. Results of testing the segmentation algorithm on model data 
The $11^{\text {th }}$ IEEE International Conference on Intelligent Data Acquisition and Advanced Computing Systems: Technology and Applications 22-25 September, 2021, Cracow, Poland

For the robot automatic pass and motion planning, a number of assumptions and limitations has been made for both the model of the coating process and the technological parameters of the process. Since the working tool of the manipulator is a movable plasma source (the microplasmatron) that forms a plasma jet with a flow of coating particles, it was assumed that the plasma jet can be simulated by a cone-shaped flow of particles. It was also assumed that the distribution of the particle flux through the plane perpendicular to the axis of the deposition cone is radially symmetric.

Two mandatory requirements were imposed on the path of the working tool of the manipulator: 1) the constancy of the distance from the working tool to the surface throughout the path and 2) the perpendicularity of the axis of the spray cone of the treated surface at any time. When these requirements are met, the path of the working tool in space will correspond to a curve on the treated surface, which is the locus of the points of intersection of the spraying cone axis with the surface. Hereinafter, this curve on the treated surface will be called "the trace of the working tool". For any point of the trace, it is easy to find the corresponding point of the actual spatial trajectory of the center of working tool. To do this, a normal to the surface at a given point of the tool trace must be built. The corresponding trajectory point will lie on this normal at a specified distance from the origin point of the trace. AS language program describes the path of the working tool as a sequence of line segments and circular arcs. Thus, the process of automatic generation of the robot manipulator program is divided into three stages. At the first stage, the path of the center of the manipulator working tool is determined as a space curve. At the second stage, this curve is approximated by a sequence of geometric primitives (line segments and arcs). At the third stage, the sequence of geometric primitives is translated into the AS program.

To generate the path of working tool center (first stage) a number of equidistant curves on the surface are build, this represents the trace of the working tools for the generated manipulator program. Each of these curves corresponds to a so-called "working segment" of an actual working tool path. Each two adjacent working segments are connected by the so-called "maneuver segment", and together they form a U-shaped fragment of the working tool path. The family of equidistant curves on the surface is generated by translation of single starting path on the surface; therefore the selection of starting path and distance between neighbor traces determines the total path of the manipulator working tool. In order to form the starting fragment of the trajectory, a specially selected geodetic line on the surface is used. In general, a geodetic line connecting two points on a surface is the shortest curve of all curves on the surface passing through those two given points. If the curve on the surface is the trace of the plasma torch nozzle, then in the case when

the geodetic curvature of the trace is zero, the sprayed material will be distributed evenly on both sides of the trajectory of the trace.

Thus, the optimal choice of the starting segment of the trajectory is to select one of the geodetic lines on the surface. It is advisable to select the starting segment so that the path segments obtained by displacing the starting segment have as little geodetic curvature as possible. We tried three ways to generate a starting fragment: two automatic and one semi-automatic. The first automatic method was based on the criterion of the minimum height proposed in [24]. A second method was developed by the research team, based on enumerating the directions of the starting segment and assessing the quality of the generated trajectories by the integral criterion. The third method also developed by the research team was semi-automatic, in which the operator sets the point and direction of the starting segment in geodetic curve. To perform the second stage of program generation procedure - an approximation of working tool path by sequence of geometric primitives, a CAL algorithm (chord and arc length algorithm) is applied to the output of the first stage. The CAL algorithm approximates an arbitrary spatial curve with a broken line, so that the specified criterion of approximation accuracy (the maximum distance from the segment of the broken line to the curve) is met. A detailed description of the CAL algorithm for the approximation of spatial curves, together with a proof of the convergence of the iterative procedure of the algorithm, can be found in [25].

The CAL algorithm was adapted to solve the main problem of this research, i.e. to make the approximation of the trajectory by a smooth curve, which is a sequence of line segments and arcs of a circle. To do this, it is enough to conjugate adjacent segments of the polyline with arcs. The simple procedure of selection of radius of conjugation arc for two adjustment segments of broken line can be briefly described as follows: choose maximum value that still meets given criteria of approximation accuracy. The choice of the radius of the conjugate arc is a compromise solution. Also, the smallest possible radius must be selected and the highest radiusto make the trajectory as smooth as possible, for every broken line vertex. It must be highlighted that fora given vertex of a broken line, the radius of the conjugate arc cannot exceed $R_{\max }(2)$ :

$$
\begin{aligned}
R_{\max } & =\min \left\{l_{1}, l_{2}\right\} \cdot \operatorname{ctg}(\varphi / 2) \\
\mathrm{R}_{\max } & =\min \left\{\mathrm{l}_{1}, \mathrm{l}_{2}\right\} \cdot \operatorname{ctg}\left(\frac{\varphi}{2}\right),
\end{aligned}
$$

where $l_{1}$ and $1 l_{2}$ are the lengths of adjacent segments of the polyline and $\varphi \varphi$ is the angle between these segments (see Fig. 4).

For a given radius $R$, the approximation error is given by the formula (3)

$$
\delta(\mathrm{R})=\frac{\left(1-\cos \left(\frac{\varphi}{2}\right)\right)}{\cos \left(\frac{\varphi}{2}\right)} \cdot \mathrm{R}
$$


The $11^{\text {th }}$ IEEE International Conference on Intelligent Data Acquisition and Advanced Computing Systems: Technology and Applications 22-25 September, 2021, Cracow, Poland

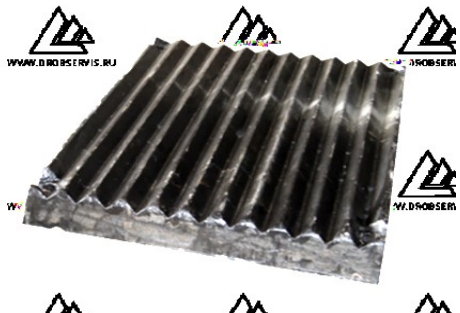

a)

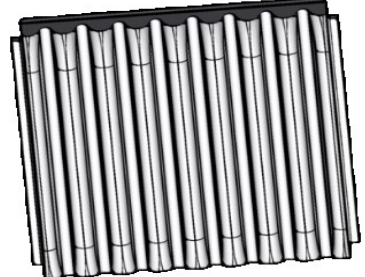

b)

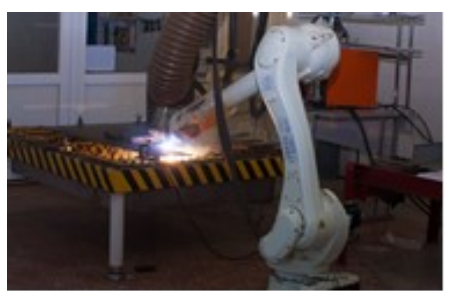

c)

Figure 4. Testing the developed algorithms at the production site: a) the crushing plate of a jaw crusher b) its 3D model, obtained as a result of robotic 3D scanning; and c) the process of robotic microplasma spraying of a Cr-based powder coating onto the crushing plate (c)

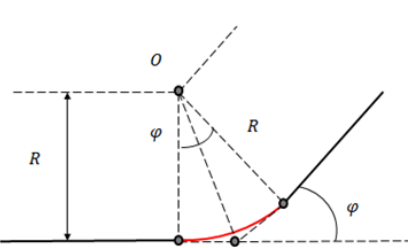

Figure 5. Conjugate line segments with a circular arc

Thus, as a compromise solution, the algorithm for choosing the radius of the mating circle was selected. At each vertex of broken line, the value of $R_{\max } \mathrm{R}_{\max }$ is calculated by the formula (1), then the corresponding error $\delta_{\mathrm{Rmax}}=$ $\delta\left(\mathrm{R}_{\max }\right)$ is calculated. Then $\delta_{\mathrm{Rmax}}$ is compared with a predetermined approximation error threshold $\delta_{\text {max }}$. In the case of $\delta_{\mathrm{R} \max } \leq \delta_{\max }, \mathrm{R}_{\max }$ is selected as the radius of the mating circle. Otherwise, the radius of the conjugate arc is calculated using equation (4)

$$
\mathrm{R}=\frac{\cos \left(\frac{\varphi}{2}\right)}{\left(1-\cos \left(\frac{\varphi}{2}\right)\right)} \cdot \delta_{\max }
$$

The described algorithm, despite a number of attractive qualities such as low computation cost and guaranteed approximation accuracy has one obvious disadvantage which it can (in some cases) introduce approximation errors that could be (in principle) avoided. For example, if input curve is an arc of circle, then algorithm would not approximate it to a single arc, but result of approximation would be a sequence of several conjugated line segments. Fromauthors' opinion, the use of pattern recognition technique applied to segmentations' task and classification of spatial curves will give prospects for improving the method of curve approximation by a sequence of geometric primitives. The research team is currently working in this direction. Software implementation of the third stage (the translation of the sequence of geometric primitive into the sequence of AS language commands) is straightforward, and will not be discussed here.

The developed method of automatic manipulator program generation was tested and the robotic microplasma spraying of a wear-resistant Cr-based coating was performed on the worn parts of the jaw's crushing plate for crushing mineral raw materials (Fig. 5). The movable plate of a jaw crusher is a large-sized part weighing $295 \mathrm{~kg}$ made of Hadfield steel (the mangalloy) with dimensions: $900 \times 820 \times 100 \mathrm{~mm}^{3}$. The plate has a ribbed surface with a distance of $100 \mathrm{~mm}$ between the centers of the ribbed projections, and the height of the projections is $30 \mathrm{~mm}$ (Fig. 5a). A robotic scanning of the plate was carried out, and then a robotic microplasma spraying of a protective coating on the worn-out sections of the plate was performed. The powder of Cr-based composite alloy AN-35 (GOST 2144875) was used for the microplasma spraying. The microplasma spraying was carried out by moving the robot arm with the microplasmatron installed on it in accordance with the obtained 3D-model of the plate (Fig. 5b). The spraying distance was maintained equal to $400 \mathrm{~mm}$. This spraying parameter was chosen based on the requirements for the structure of the coating.

Production tests of the crushing plate were carried out under conditions of grinding polymetallic ores of various hardness with continuous operation of the crusher for at least 30 minutes with a loading from $70 \%$ to $100 \%$ of the height of the crushing chamber. The movable plate of the jaw crusher, coated after its main resource had been depleted, was additionally operated for 6 months, being subjected to the optimal load for 5-6 hours per shift. Thus, the plate's service life was extended with the same crusher performance. A production test certificate was received (Ust-Kamenogorsk, Kazakhstan), and it was concluded that the service life of the movable plate of the jaw crusher restored by robotic plasma spraying of the $\mathrm{Cr}$ - based protective coating was increased by $15 \%$ compared to the plates not subject to restoration.

\section{CONCLUSION}

Algorithms have been developed for automatic path and motion planning for a robot manipulator performing microplasma spraying of coatings, the program for its movement along a 3D model reconstructed from the data of robotic 3D scanning of a plasma-processed large-sized product has been automatically generated.

The pass planning algorithm is based on the selection of the starting segment of the trajectory as a geodetic line on the surface. An algorithm has been developed for optimal curve approximation by a sequence of geometric primitives such as segmented lines and arcs, by providing 
conjugations of curve sections. The new motion planning algorithm ensures smooth movement of the robotmanipulator working tool at a constant modulus speed along a 3D model of the product, without the risk of unwanted large values of centripetal acceleration during manipulator maneuvers. A practical implementation of the developed algorithms for the surface segmentation and automatic generation of a robot manipulator program was carried out, with the help of an intelligent system based on an industrial robot Kawasaki where a microplasma spraying of a protective coating was carried out on a jaw crusher plate, which was then operated for 6 months to crush mineral raw materials.

The developed new algorithms for an intelligent robotic system performing plasma spraying of coatings onto surfaces with complex shape are of interest to a wide range of researchers in the field of robotics and automation of production areas with mechatronic systems.

Further development of research involves comparing the final product quality produced with different robot control algorithms for evaluation of methods and results using the spraying simulator.

\section{REFERENCES}

[1] W. Chen, J. Liu, Y. Tang, and H. Ge, “Automatic Spray Trajectory Optimization on Bézier Surface," Electronics, vol. 8 (168), pp. 116, February 2019, DOI: 10.3390/electronics8020168.

[2] I. Karymsakova, N. Denissova, S. Kumargazhanova et al. "Robotic Plasma Spraying System For Implants of Complex Structure: 3D model and motion planning," International Journal of Computing, vol. 19(2), pp. 224-23, June 2020, DOI: https://doi.org/10.47839/ijc.19.2.1765.

[3] Duro R., et al (Eds). Advances in Intelligent Robotics and Collaborative Automation. River Publishers, Gistrup, 2015. doi: https://doi.org/10.13052/rp-9788793237049

[4] D. McMorran, D.C.K. Chung, J. Li, M. Muradoglu, O.W. Liew, and T.W. Ng, "Adapting a Low-Cost Selective Compliant Articulated Robotic Arm for Spillage Avoidance," Journal of Laboratory Automation, vol. 21, pp. 799-805, February 2016, DOI: https://doi.org/10.1177/2211068216630742.

[5] P. Zhang, J. Gong, and L. Wei, "Arc Spray Gun New Models of Considering the Flow in the Process of Spraying for Spray-Painting Robot," IEEE International Conference on Intelligent Human Machine Systems and Cybernetics, vol. 01, pp. 165-168, August 2015, DOI: https://doi.org/10.1109/IHMSC.2015.41.

[6] C. Feng, Y. Xiao, A. Willette, W. McGee, and V. R. Kamat, "Vision guided autonomous robotic assembly and as-built scanning on unstructured construction sites," Automation in Construction, vol. 59, pp. 128-138, July 2015, DOI: https://doi.org/10.1016/j.autcon.2015.06.002.

[7] R.C. Tucker, Jr., ed., Introduction to Coating Design and Processing, Thermal Spray Technology, ASM Handbook, Ohio: ASM international, vol. 5A, pp. 76-88, 2013, DOI: https://doi.org/10.31399/asm.hb.v05a.a0005725.

[8] B. Fotovvati, N. Namdari, and A. Dehghanghadikolaei, "On Coating Techniques for Surface Protection: A Review," Journal of Manufacturing and Materials Processing, vol. 3, pp. 1-22, March 2019, DOI:10.3390/jmmp3010028.

[9] G. Trigatti, P. Boscariol, L. Scalera, D. Pillan, and A. Gasparetto, "A new path-constrained trajectory planning strategy for spray painting robots - rev.1," The International Journal of Advanced Manufacturing Technology, vol. 98, pp. 2287-2296, October 2018, DOI: https://doi.org/10.1007/s00170-018-2382-2.
[10] W. Chen, Y. Tang, and Q. Zhao, "A novel trajectory planning scheme for spray painting robot with Bézier curves," IEEE Control and Decision Conference, pp. 6746-6750, May 2016, DOI:10.1109/CCDC.2016.7532211.

[11] W. Chen, C. Sun, H. Liu, J. Liu, and Y. Tang, "Path planning scheme for spray painting robot with Bézier curves on complex curved surfaces," IEEE Youth Academic Annual Conference of Chinese Association of Automation (YAC), pp. 698-703, May 2017, DOI: 10.1109/YAC.2017.7967499.

[12] Y. Zhoua, S. Ma, A. Li, and L. Yang, "Path Planning for Spray Painting Robot of Horns Surfaces in Ship Manufacturing," $3^{\text {rd }}$ IOP Conf. Ser.: Mater. Sci. Eng., pp.1-7, May 2019, DOI:10.1088/1757899X/521/1/012015.

[13] Z. Fu, B. Xiao, C. Wu, and J. Yang, "A genetic algorithm-based surface segmentation method for spray painting robotics," IEEE Control and Decision Conference, pp. 4049-4054, May 2017, DOI:10.1109/CCDC.2017.7979209.

[14] B. Zhou, F. Fang, S. Zhenhua, M. Zhengda, and D. Xianzhong, "Fast and templatable path planning of spray painting robots for regular surfaces," IEEE Control Conference, pp. 5925-5930, July 2015, DOI:10.1109/ChiCC.2015.7260567.

[15] Y. Zeng, Y. Zhang, J. He, et al. "Prediction Model of Coating Growth Rate for Varied Dip-Angle Spraying Based on Gaussian Sum Model," Mathematical Problems in Engineering, vol. 2016, pp. 1-7, January 2016, DOI:10.1155/2016/9369047.

[16] Y. Kondratenko, et al. "Slip Displacement Sensors For Intelligent Robots: Solutions and Models," 2013 IEEE 7th Int. Conf. on Intelligent Data Acquisition and Advanced Computing Systems (IDAACS), Berlin, vol. 2, pp. 861-866, September, 2013, DOI: 10.1109/IDAACS.2013.6663050

[17] Y. Kondratenko, O. Gerasin, A. Topalov, "A simulation model for robot's slip displacement sensors," International Journal of Computing, Vol.15, Issue 4, pp. 224-236, 2016, http://www.computingonline.net/computing/article/viewFile/854/7 68

[18] A.N. Tkachenko., et al. "Evolutionary adaptation of control processes in robots operating in non-stationary environments," Mechanism and Machine Theory, vol. 18, no. 4, pp. 275-278, 1983, DOI: 10.1016/0094-114X(83)90118-0

[19] M. Patil, T. Abukhalil, S. Patel, T. Sobh, "UB SWARM: hardware implementation of heterogeneous swarm robot with fault detection and power management," International Journal of Computing, vol 15(3), pp. 162-176, 2016, https://doi.org/10.47839/ijc.15.3.849

[20] AS Language Reference Manual. Kawasaki Robot Controller E Series, Kawasaki Heavy Industries, Ltd, 2015, 548 p.

[21] D. Alontseva, E. Ghassemieh, S. Voinarovych, et al.: "Manufacturing and characterization of robot assisted microplasma multilayer coating of Titanium implants," Johnson Matthey Technol. Rev., vol. 64(2), pp. 180-191, 2020, https://doi.org/10.1595/205651320X15737283268284

[22] D.L. Alontseva, E. Ghassemieh, A.L. Krasavin, and A.T. Kadyroldina, "Development of 3D Scanning System for Robotic Plasma Processing of Medical Products with Complex Geometries," Journal of Electronic Science and Technology, vol. 18(3), pp. 212-222, September 2020, DOI: https://doi.org/10.1016/j.jnlest.2020.100057.

[23] D. Alontseva, A. Krasavin, A. Kadyroldina, A. Kussaiyn-Murat, "Segmentation Algorithm for Surface Reconstruction According to Data Provided by Laser-Based Scan Point," Communications in Computer and Information Science, vol. 998, pp. 1-10, January 2019, DOI:https://doi.org/10.1007/978-3-030-12203-4_1.

[24] W. Chen, D. Zhao, "Path Planning for Spray Painting Robot of Workpiece Surfaces," Mathematical Problems in Engineering, vol. 2013, pp. 1-6, August 2013, DOI:http://dx.doi.org/10.1155/2013/659457.

[25] J.A. Horst, I. Beichl, "Efficient piecewise linear approximation of space curves using chord and arc length," Proceedings of the SME Applied Machine Vision '96 Conference, Cincinnati Ohio, pp. 112, June 1996. 